\title{
NOTE ON A PARTITION LIMIT THEOREM FOR RANK AND CRANK
}

\author{
PERSI DIACONIS, SVANTE JANSON, AND ROBERT C. RHOADES
}

\begin{abstract}
If $\lambda$ is a partition of $n$, the rank $r k(\lambda)$ is the size of the largest part minus the number of parts. Under the uniform distribution on partitions, in Bringmann-MahlburgRhoades 4 it is shown that $r k(\lambda) / \sqrt{6 n}$ has a limiting distribution. We identify the limit as the difference between two independent extreme value distributions and as the distribution of $\beta(T)$ where $\beta(t)$ is standard Brownian motion and $T$ is the first time that an independent three-dimensional Brownian motion hits the unit sphere. The same limit holds for the crank.
\end{abstract}

Let $\lambda=\left(\lambda_{1}, \lambda_{2}, \ldots, \lambda_{l}\right)$ with $\lambda_{1} \geq \lambda_{2} \geq \cdots \geq \lambda_{l}>0, \sum_{i=1}^{l} \lambda_{i}=n$ be a partition of $n$. The rank $r k(\lambda)=\lambda_{1}-l$ was introduced by Dyson [7 to explain some of Ramanujan's congruences (e.g., $p(5 n+4) \equiv 0(\bmod 5))$. This story with its many developments to other primes and cranks can be accessed from the Wikipedia entry on Ramanujan's congruences and its references; see, for example, [1] for more on the crank. In [4, 5], Bringmann, Mahlburg, and Rhoades studied the moments of $r k(\lambda)$ when $\lambda$ is chosen from the uniform distribution on the partitions of $n, \mathcal{P}(n)$. Write $p(n)$ for $|\mathcal{P}(n)|$. Their results imply

Theorem $0.1([4])$. There is a distribution function $F_{r}(x)$ on $\mathbb{R}$ such that, for all $x$,

$$
\lim _{n \rightarrow \infty} \frac{1}{p(n)}\left|\left\{\lambda \in \mathcal{P}(n): \frac{r k(\lambda)}{\sqrt{6 n}} \leq x\right\}\right| \longrightarrow F_{r}(x) .
$$

The main result of this note identifies the limit $F_{r}(x)$. Recall the extreme value distribution function $F_{e}(x)=e^{-e^{-x}},-\infty<x<\infty$. This occurs as the limiting distribution of the maximum of a wide variety of sequences of random variables. Indeed, Erdös and Lehner [8] proved that it governs the distribution of the largest part of a random partition:

$$
\lim _{n \rightarrow \infty} \frac{1}{p(n)}\left|\left\{\lambda \in \mathcal{P}(n): \frac{\lambda_{1}-\frac{\sqrt{6 n}}{\pi} \log (\sqrt{6 n} / \pi)}{\sqrt{6 n} / \pi} \leq x\right\}\right| \rightarrow F_{e}(x) .
$$

By reflection symmetry, the limiting distribution of $l(\lambda)$ is the same. This makes it plausible that the limiting distribution of $r k(\lambda)$ should be the difference between two independent extreme value distributions. This is our result:

Proposition 0.2. The limit $F_{r}(x)$ in (10.1) is the distribution function of $\left(W_{1}-W_{2}\right) / \pi$ where $W_{i}$ are independent with distribution function $F_{e}(x)$. Thus $F_{r}(x)=1 /\left(1+e^{-\pi x}\right)$.

Remark. (1) From (0.2) both $\lambda_{1}$ and $l(\lambda)$ are typically of size $\sqrt{n} \log n$. At first it seems surprising that $r k(\lambda)$ is only of size $\sqrt{n}$. On reflection, we see that the additive $\sqrt{n} \log n$ terms cancel in $\lambda_{1}-l$ and the scaling determines the size. The limit in the proposition is exactly the convolution of the two fluctuation distributions. Indeed,

Date: November 4, 2018. 
Szekeres [11] has proved a local limit theorem for the joint distribution of $\lambda_{1}$ and $l(\lambda)$ which shows that they are asymptotically independent.

(2) Let $N(n, m)$ be the number of partitions of $n$ with rank equal to $m$. Let $N_{k}(n)=$ $\sum_{m \in \mathbb{Z}} m^{k} N(n, m)$ be the $k$ th moment. Then $N_{0}(n)=p(n), N_{k}(n)=0$ for $k$ odd by symmetry $(N(n, m)=N(n,-m))$. Bringmann, Mahlburg, and the third author [4] prove that, as $n$ tends to infinity, for each $l=1,2, \ldots$,

$$
\frac{N_{2 l}(n)}{(6 n)^{l} p(n)} \sim\left(2^{2 l}-2\right)\left|B_{2 l}\right|
$$

where $B_{2 l}$ is the $2 l$ th Bernoulli number, $\frac{t}{\left(e^{t}-1\right)}=\sum_{n=0}^{\infty} B_{n} t^{n} / n$ !. A routine application of the method of moments yields (0.1). They also proved that the crank statistic has the same limiting moments. Thus the limit theorem and proposition apply to the crank.

A web search for "Bernoulli numbers as moments" leads to work of Pitman and Yor [9]. They show the following: let $\beta(t)$ be standard one-dimensional Brownian motion. Let $T$ be the first time that standard three-dimensional Brownian motion hits the unit ball ( $T$ is independent of $\beta(t)$ ). Then $\beta(T)$ has odd moments zero and

$$
E\left(\beta(T)^{2 l}\right)=\left(2^{2 l}-2\right)\left|B_{2 l}\right| .
$$

This shows that $F_{r}(x)=P\{\beta(T) \leq x\}$. This strains intuition; what is the connection between Brownian motion and partitions? Our proposition explains things by showing how $F_{r}(x)$ has other probabilistic interpretations.

(3) A simple calculation shows that the sth absolute moment of the logistic limit distribution $F_{r}$ is $2 \Gamma(s+1) \pi^{-s}\left(1-2^{1-s}\right) \zeta(s)$, for any $s>0$. It follows, since uniform integrability is implied by (ii), that

$$
\frac{\sum_{m}|m|^{s} N(n, m)}{(6 n)^{s / 2} p(n)} \sim 2 \Gamma(s+1) \pi^{-s}\left(1-2^{1-s}\right) \zeta(s) .
$$

For odd integers $s$ this was recently obtained, with an error term, via other methods described in a forthcoming work by Bringmann and Mahlburg [6].

Proof of Proposition [0.2. From [9],

$$
E\left(e^{i t \beta(T)}\right)=\frac{t}{\sinh (t)} .
$$

If $W$ has distribution function $e^{-e^{-t}}$ and so density $e^{-e^{-t}-t}$ on $\mathbb{R}$,

$$
E\left(e^{i t W}\right)=\int_{-\infty}^{\infty} e^{i t x-e^{-x}-x} d x=\int_{0}^{\infty} y^{-i t} e^{-y} d y=\Gamma(1-i t) .
$$

Thus $E\left(e^{i t\left(W_{1}-W_{2}\right)}\right)=\Gamma(1-i t) \Gamma(1+i t)=i t \Gamma(i t) \Gamma(1-i t)=\frac{i t \pi}{\sin (i t \pi)}=\frac{t \pi}{\sinh (t \pi)}$. Here, the reflection formula $\Gamma(z) \Gamma(1-z)=\pi / \sin (\pi z)$ is used. The distribution of $\left(W_{1}-W_{2}\right) / \pi$ thus equals the distribution of $\beta(T)$. It is well known and easy to verify that the difference between two independent extreme value variates has the logistic distribution as stated. 
Remark. One may also study the limiting distribution of $r k(\lambda)$ using the conditioned limit approach of Fristedt [3], [10], or the method of Corollary 2.6 of Borodin [2]. These avoid moments and give the limiting distribution of many further statistics. Fristedt's approach may be developed to prove the asymptotic independence of $\lambda_{1}$ and $l(\lambda)$. Combining this, the results of Erdös-Lehner, and the calculations of this note gives an alternate proof of the main theorem.

\section{REFERENCES}

[1] G. E. Andrews, and F. G. Garvan, Dyson's crank of a partition. Bull. Amer. Math. Soc. (N.S.) 181988 No. 2, 167-171.

[2] A. Borodin, Periodic Schur process and cylindric partitions. Duke Math. J., 140 no. 3 2007, 391-468.

[3] B. Fristedt, The structure of random partitions of large integers. Trans. Amer. Math. Soc., 337 no. 2, 1993 703-735.

[4] K. Bringmann, K. Mahlburg, and R.C. Rhoades, Asymptotics for rank and crank moments. Bull. Lond. Math. Soc., 43 no. 4, 2011, 661-672.

[5] K. Bringmann, K. Mahlburg, and R.C. Rhoades, Taylor coefficients of mock-Jacobi forms and moments of partition statistics. to appear, Math. Proc. Cambridge Phil. Soc..

[6] K. Bringmann and K. Mahlburg, Asymptotic inequalities for positive crank and rank moments. preprint 2012.

[7] F. J. Dyson, Some guesses in the theory of partitions. Eureka, Cambridge 8 1944 10-15.

[8] P. Erdös and J. Lehner, The distribution of the number of summands in the partitions of a positive integer, Duke Math. J., 8 (1941) 335-345.

[9] J. Pitman and M. Yor, Infinitely divisible laws associated with hyperbolic functions. Canad. J. Math. 55 (2003) No. 2, 292-330.

[10] B. Pittel, On a likely shape of the random Ferrers diagram. Adv. in Appl. Math., 18 No. 4, 1997, $432-488$.

[11] G. Szekeres, Asymptotic distribution of partitions by number and size of parts. Number Theory, Vol. I (Budapest, 1987), Colloq. Math. Soc. János Bolyai 51527 - 538. North-Holland, Amsterdam, 1990.

Stanford University, Department of Mathematics and Statistics, Sequoia Hall, 390 Serra MaLl, Stanford, CA 94305-4065, USA

E-mail address: diaconis@math.stanford.edu

Uppsala University, Department of Mathematics, Box 480, 751 06, Uppsala, Sweden

E-mail address: svante.janson@math.uu.se

Stanford University, Department of Mathematics, Sloan Hall, 450 Serra Mall, Stanford, CA 94305-2125, USA

E-mail address: rhoades@math.stanford.edu 